\title{
BETTER INTEGRATED INSTRUCTION OF GEOMETRY AND ALGEBRA IN PRIMARY EDUCATION
}

\author{
Metodi Glavche $^{1}$, Katerina Anevska ${ }^{2}$, Risto Malčeski $^{3}$
}

\begin{abstract}
The integrated teaching of mathematics is important for the development of the students from every aspect. Practice shows that integrated knowledge improves the quality of thinking and at the same time enables the students to acquire permanent and applicable knowledge. Although there are declarative efforts for acquiring integrated knowledge, there is lack of inter-subject and intra-subject integration. This paper scrutinizes how elementary geometry knowledge can be used to improve integrated instruction. We are going to show how to come up with formulas for some sums of numbers by using areas of squares and rectangles. The latter is especially important since these formulas are presented to the students either in a dogmatic way, or through mathematic induction, which is incomprehensible for most students of this age.
\end{abstract}

\section{INTRODUCTION}

The aim of the integration of the education is to present the young generations with a complete and comprehensive image of nature, society and their roles. On the other hand, the traditional division of the content into separate independent subjects aims for the students to acquire profound knowledge from specific areas, which they will subsequently be able to connect in a whole. This process can be stimulated by successful inter-subject and intrasubject integration of the mathematics instruction.

The intra-subject integration can vary from partial to complete. It should also promote integral learning of mathematical content, which should result in applicable knowledge and skills. 
Bearing all this in mind, we support the view that the intra-subject integration of mathematics should be taken seriously, and that it is very important to implement it as much as possible when it comes to operational knowledge.

The knowledge about the area of a square and a rectangle which can be used for devising formulas for calculating some sums is especially useful for the improvement of the intra-subject integration.

Although the practice shows that it is very important for the development of the quality of thinking, it is not sufficiently implemented in the education system yet. As far as the integration of the instruction in primary education is concerned, it is still at an elementary level although there are new syllabi, textbooks, and other options, such as in [1], [3] and [4]. The integration of the instruction can improve the following aspects of the quality of thinking:

- The elasticity of thinking, which is significantly improved when learning integrated mathematical content,

- The adequate thinking patterns which increase the ability to make a good selection of methods and means for solving specific problems when learning integrated content,

- The rationality of thinking, which is directly related to the adequate thinking patterns,

- The depth of thinking, which is especially important in the integrated learning of the content because it improves the ability of the students to analyze the problems from all aspects and to extend the use of the knowledge, and

- It decreases patterned thinking that is accompanied by the so-called "functional stability effect", according to which the object is used only in its given form.

\section{INTEGRATION OF THE INSTRUCTION BY CALCULATING SUMS WITH THE USE OF GEOMETRIC FIGURES}

The integration of the mathematics instruction can be achieved in various ways, although it seems that it is most successful when the elementary knowledge about the areas of squares and rectangles is used for acquiring formulas for calculating some sums of numbers. This is very important since most of these formulas are presented to the students either in a dogmatic way, or through mathematical induction, which is incomprehensible for most students of this age. We are going to show several examples. 
Example 1. The primary school students learn the formula for calculating the sum of the first $n$ natural numbers by using the pattern which is historically linked to K. F. Gauss usually in this way:

$$
\begin{aligned}
& S=1+2+3+\ldots+(n-2)+(n-1)+n \\
& \begin{aligned}
2 S= \\
= \\
=
\end{aligned} \\
& =\underbrace{(1+n)+(1+2+3+\ldots+(n-2)+(n-1)+n]+[n+(n-1)+(n-2)+\ldots+3+2+1]}_{n} \\
& S=1+2+3+\ldots+(n-2)+(n-1)+n=\frac{n(n+1)}{2} .
\end{aligned}
$$

We can also calculate the sum of the first $n$ natural numbers with the help of Figure 1.

Hence, we have $n$ rectangles with width 1 and lengths $1,2,3, \ldots n$ respectfully. The area of the figure $A B F E$ is equal to $S$. We add the figure $C D E F$ to $A B F E$ and the result is $A B C D$ consisting of $n$ matching rectangles with sides 1 and $n+1$. It has an area $n(n+1)$, and

$$
P_{A B C D}=2 P_{A B F E}=2 S,
$$

thus $S=\frac{n(n+1)}{2}$. Hence we got the formula in another way (1).

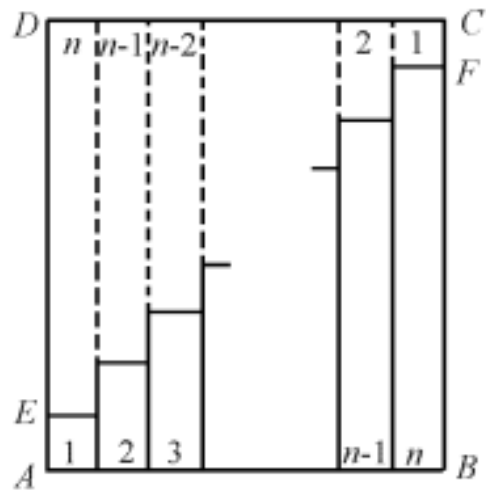

Figure 1

Example 2. The formula for the sum of the first $n+1$ odd numbers can be acquired analogously to example 1 in the following way:

$$
\begin{aligned}
S= & 1+3+5+\ldots+(2 n-3)+(2 n-1)+(2 n+1) \\
2 S & =[1+3+\ldots+(2 n-1)+(2 n+1)]+[(2 n+1)+(2 n-1)+\ldots+3+1] \\
& =\underbrace{2(n+1)+2(n+1)+\ldots+2(n+1)+2(n+1)}_{n+1}=2(n+1)^{2} \\
S & =1+3+5+\ldots+(2 n-3)+(2 n-1)+(2 n+1)=(n+1)^{2} .
\end{aligned}
$$

The Sum $S$ can also be calculated by using geometric figures. The lengths of the squares $A C F E$ and $A C^{\prime} F^{\prime} E^{\prime}$ are $k$ and $k+1$ (Figure 2). The difference between these two squares is the figure $C C^{\prime} F^{\prime} E^{\prime} E F$, which is called gnomon, and consists of one square with an area equal to

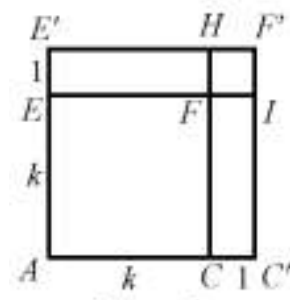

Figure 2 
1 and two rectangles with areas $k$. Accordingly, if we add the gnomon $C C^{\prime} F^{\prime} E^{\prime} E F$ with an area $2 k+1$ to the square $A C F E$ with an area $k^{2}$, the result is $A C^{\prime} F^{\prime} E$ with an area $(k+1)^{2}$.

Now we are going to analyze the square $A C^{\prime} F^{\prime} E^{\prime}$ with side 1 to which gnomons with areas of

$$
2 \cdot 1+1=3,2 \cdot 2+1=5,2 \cdot 3+1=7, \ldots, 2 n+1
$$

are added (Figure 3 ). In this way, we got the square $A C F E$ with side $n+1$ and area $(n+1)^{2}$. On the other hand, the area of the square $A C F E$ is equal to the sum of the areas of the square $A C^{\prime} F^{\prime} E^{\prime}$ and the added gnomons, and thus it is equal to $1+3+5+7+\ldots+(2 n+1)$. This way we proved that the formula is correct (2).

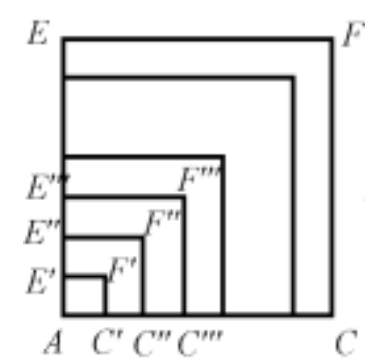

Figure 3

Example 3. In most of the cases the teachers present the gifted students in primary school with the formula

$$
1 \cdot 2+2 \cdot 3+3 \cdot 4+\ldots+n(n+1)=\frac{n(n+1)(n+2)}{3}
$$

in a dogmatic way. In some cases, the teachers try to prove it by using the principle of mathematical induction, although there is no need for this. This formula can be acquired by using the rectangle presented on Figure 4. Thus Figure 4 and example 1 allow us to conclude that the

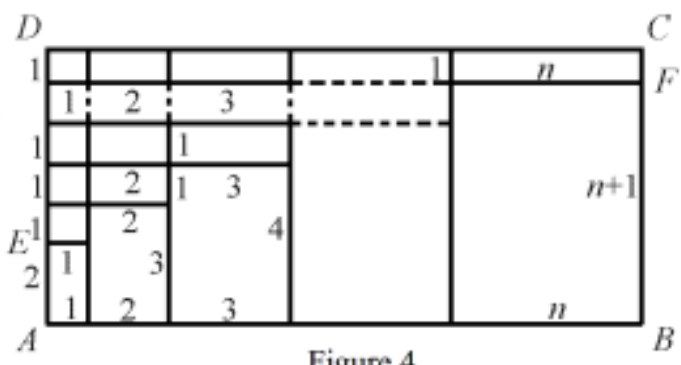

Figure 4 lengths of the rectangle are $\overline{B C}=n+2$ and

$$
\overline{A B}=1+2+\ldots+n=\frac{n(n+1)}{2},
$$

meaning that the area is

$$
P_{A B C D}=\frac{n(n+1)(n+2)}{2} .
$$

Further on, the line $E F$ separates the rectangle into two parts - the figure $A B F E$, which consists of rectangles with areas $1 \cdot 2,2 \cdot 3,3 \cdot 4, \ldots, n(n+1)$ and

$$
P_{A B F E}=1 \cdot 2+2 \cdot 3+3 \cdot 4+\ldots+n(n+1) \text {. }
$$


And the figure $C D E F$, which is located on the upper part of the rectangle consisting of $n$ rows with areas $1,1+2,1+2+3, \ldots, 1+2+3+\ldots+n$. If we first use the formula (1), and then the formula (6), we get

$$
\begin{aligned}
P_{C D E F} & =1+(1+2)+(1+2+3)+\ldots+(1+2+3+\ldots+n) \\
& =\frac{1 \cdot 2}{2}+\frac{2 \cdot 3}{2}+\frac{3 \cdot 4}{2}+\ldots+\frac{n(n+1)}{2} \\
& =\frac{1}{2}[1 \cdot 2+2 \cdot 3+3 \cdot 4+\ldots+n(n+1)]=\frac{1}{2} P_{A B E F} .
\end{aligned}
$$

But $P_{A B C D}=P_{A B F E}+P_{C D E F}$ and if we use the equations (4), (5) and (6) we get

$$
\begin{aligned}
\frac{n(n+1)(n+2)}{2} & =P_{A B C D}=P_{A B F E}+P_{C D E F} \\
& =P_{A B F E}+\frac{1}{2} P_{A B F E}=\frac{3}{2} P_{A B F E} \\
& =\frac{3}{2}[1 \cdot 2+2 \cdot 3+3 \cdot 4+\ldots+n(n+1)],
\end{aligned}
$$

thus resulting in the formula (3).

The next example shows how the formula designed in example 3 can be used for calculating the sum of the squares of the first $n$ natural numbers. We also need to mention that the primary school students already have the required knowledge for cognitive use of this frequently used formula.

Example 4. From the equation $k^{2}=k(k+1)-k$ follows that $k=1,2, \ldots, n$ resulting in the equations

$$
\begin{aligned}
& 1^{2}=1(1+1)-1=1 \cdot 2-1 \\
& 2^{2}=2(2+1)-2=2 \cdot 3-1 \\
& \ldots \ldots \ldots \ldots \ldots \ldots \ldots \ldots \ldots \ldots \ldots \ldots \ldots \ldots \ldots \ldots \ldots \ldots \ldots \ldots \\
& n^{2}=n(n+1)-n .
\end{aligned}
$$

If we perform the operation addition on the left and right sides of the last equations and use the formulas (1) and (3) we get the following formula for the sum of the squares of the first $n$ natural numbers:

$$
\begin{aligned}
1^{2}+2^{2}+\ldots+n^{2} & =1 \cdot 2-1+2 \cdot 3-2+\ldots+n(n+1)-n \\
& =1 \cdot 2+2 \cdot 3+\ldots+n(n+1)-[1+2+\ldots+n] \\
& =\frac{n(n+1)(n+2)}{3}-\frac{n(n+1)}{2}=n(n+1)\left(\frac{n+2}{3}-\frac{1}{2}\right) \\
& =\frac{n(n+1)(2 n+4-3)}{6}=\frac{n(n+1)(2 n+1)}{6},
\end{aligned}
$$




$$
1^{2}+2^{2}+\ldots+n^{2}=\frac{n(n+1)(2 n+1)}{6}
$$

Example 5. The square $A B C D$ is presented in Figure 5. The length of the side is

$$
1,1+2,1+2+3, \ldots, 1+2+\ldots+(k-1) .
$$

In the square $A B C D$ we are going to construct other squares with an apex that matches the apex $A$ and sides parallel to the sides $A B$ and $A D$. The length of the sides is

$$
1,1+2,1+2+3, \ldots, 1+2+\ldots+(k-1) .
$$

The gnomon $B C D D^{\prime} C^{\prime} B$ has width $k$. Its area is

$$
\begin{aligned}
P_{B C D D^{\prime} C^{\prime} B} & =2 P_{D D^{\prime} C E}-P_{E C F C^{\prime}} \\
& =2 k(1+2+3+\ldots+k)-k^{2} \\
& =2 k \frac{k(k+1)}{2}-k^{2}=k^{3} .
\end{aligned}
$$

If we take a square and we consequently construct gnomons with widths $2,3,4, \ldots, n$, we get a square with a side

$$
1+2+\ldots+n=\frac{n(n+1)}{2},
$$

which means that its area is $\left[\frac{n(n+1)}{2}\right]^{2}$. On the other hand, the area of the square is equal to the sum of the area of the square and the areas of the gnomons with sides $2,3,4, \ldots, n$, hence (7) confirms that the formula

$$
1^{3}+2^{3}+3^{3}+\ldots+n^{3}=\left[\frac{n(n+1)}{2}\right]^{2}
$$

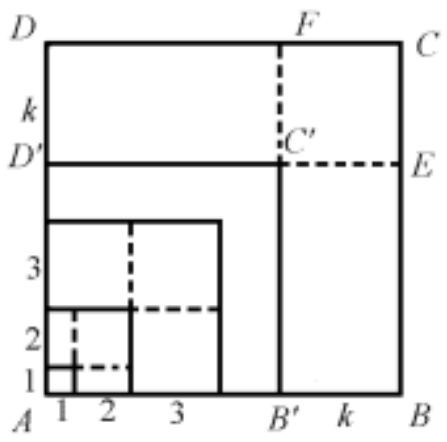

Figure 5

is correct.

\section{Conclusion}

In the previous analyses we showed several examples which proved that we can calculate some sums of natural numbers by using geometric figures. As we have previously mentioned, these and similar examples can significantly improve the integration of geometry and algebra in the primary education. It is a fact that for better integration of the instruction in the elementary and primary education we need to carry out reforms. Here are some of them: 
- The integration of the instruction should be seriously taken into consideration when creating the syllabi. It most definitely should not be just a result of the good will of the authors and teachers.

- The new textbooks must contain integrated content. These textbooks should replace the existing textbooks as soon as possible, because they have many technical content mistakes and are so elementary that they endanger the future of the next generations.

- Having in mind that the successful integration of the instruction is largely conditioned by the training of the teachers, we need to create a new generation of teachers who will be trained to fully carry out the integration of the mathematics instruction, which includes a different teacher training approach.

\section{CONFLICT OF INTEREST}

No conflict of interest was declared by the authors.

\section{Author's ConTributions}

All authors contributed equally and significantly to writing this paper. All authors read and approved the final manuscript.

\section{References}

[1] V. Gogovska, R. Malčeski, (2012). Improvement intra-disciplinary integration of mathematics instruction, Elsevier, Procedia - Social and Behavioral Sciences, 46, 5420-5424

[2] S. Grozdev, (2007). For High Achievements in Mathematics. The Bulgarian Experience (Theory and Practice). ADE, Sofia, ISBN 97895492139-1-1

[3] R. Malčeski, K. Anevska, M. Glavche, (2014). The integration of mathematics instruction in elementary education, IJSR, ISSN 2319-7064, Vol. 3, Iss. 9, pp. 2394-2398

[4] R. Malčeski, V. Gogovska, (2005). Integration of mathematics curriculum, a Challenge of the contemporary Mathematics, International Conference on Mathematics Education, 3-5 June 2005, Svishtov - Bulgaria 
[5] Р. Малчески, (2016). Методика на наставата по математика (второ издание), Сојуз на математичари на Македонија, Скопје, ISBN 9789989-646-71-3

${ }^{1)}$ Faculty of Pedagogy, Ss. Cyril and Methodius University, Skopje, Macedonia E-mail address: mglavche@gmail.com

${ }^{2)}$ Faculty of informatics, FON University, Skopje, Macedonia E-mail address: risto.malceski@gmail.com

${ }^{3)}$ Faculty of informatics, FON University, Skopje, Macedonia E-mail address: anevskak@gmail.com 